\title{
Accelerometer Static Calibration based on the PSO algorithm
}

\author{
Zhang liguo $^{1, \mathrm{a}}$, Li wenchao ${ }^{1, \mathrm{~b}}$, Liu huilian ${ }^{1}$ \\ ${ }^{1}$ Measurement Technology and Instrumentation Key lab of Hebei Province, Yanshan \\ university,Qinhuangdao 066004, China \\ azlgtime@163.com, ${ }^{b}$ tianlong6670096@163.com, 'hdhgpi@163.com
}

Keywords: accelerometer, static calibration, the PSO algorithm

\begin{abstract}
This paper introduces MEMS accelerometer static calibration method based on the PSO algorithm. According to the mathematical model of the MEMS accelerometer, the PSO algorithm is applied to estimate the mathematical model, including scale factors, bias, and installation errors. The method does not require a three-axis rotary platform, and the calculation is simple, easy to implement, high accuracy and provides a new choice for outdoor testing environment. Analyzing the experimental data, the random errors in the sampling process have been eliminated effectively, and the calibration effect is better than the commonly static correction method.
\end{abstract}

\section{Introduction}

With the increasing of MEMS manufacturing technology, MEMS devices have been widely used and are rapidly spreading, especially in autonomous navigation, motion capture and rehabilitation medical care. Among MEMS sensors, MEMS accelerometer is the most widely used.

In order to improve accelerometer's measurement accuracy, the calibration of the acceleration's output is necessary. Currently, the most common method is the maximum and minimum method, but this method can only estimate the scale factor error and bias, and is susceptible to the influence of random errors. Seong ${ }^{[1]}$, Vladimir Chapsky ${ }^{[2]}$ and Gustavo ${ }^{[3]}$ proposed the their calibration method, which increased the reliability and accuracy of the measurement. But the basic principle is similar to static calibration method.

This paper first analyzes the error sources of MEMS accelerometer, set up the trial accelerometer mathematical model, proposes the PSO static calibration method based on the ellipsoid constraint. The result shows the validity of the proposed approach.

\section{Preliminaries}

\section{The Accelerometer Static Calibration Mathematical Model}

This paper uses the trial accelerometer ADXL345 manufactured by ADI. This paper sets its measurement range for $\pm 2 \mathrm{~g}$, so $1 \mathrm{~g}=255$ Counts. The data is collected through I2C bus, sampled at $40 \mathrm{~Hz}$.

Analyzing MEMS accelerometer measurement principle and environment, we can know the main errors include three gain factors, three biases and installation errors ${ }^{[4]}$.According to the characteristics of the measurement error, the MEMS accelerometer error model in an inertial measurement unit is equation (1).

$$
\left[\begin{array}{l}
h_{x} \\
h_{y} \\
h_{z}
\end{array}\right]=\left[\begin{array}{l}
b_{x}+n_{x} \\
b_{y}+n_{y} \\
b_{z}+n_{z}
\end{array}\right]+\left[\begin{array}{ccc}
S_{x} & -a_{4} S_{y} & -a_{5} S_{z} \\
a_{1} S_{x} & S_{y} & -a_{6} S_{z} \\
-a_{2} S_{x} & a_{3} S_{y} & S_{z}
\end{array}\right]\left[\begin{array}{l}
g_{x} \\
g_{y} \\
g_{z}
\end{array}\right]=\left[\begin{array}{l}
b_{x}+n_{x} \\
b_{y}+n_{y} \\
b_{z}+n_{z}
\end{array}\right]+\left[\begin{array}{ccc}
S_{x} & K_{12} & K_{13} \\
K_{21} & S_{y} & K_{23} \\
K_{31} & K_{32} & S_{z}
\end{array}\right]\left[\begin{array}{l}
g_{x} \\
g_{y} \\
g_{z}
\end{array}\right]
$$

$h_{x}, h_{y}, h_{z}$ are the actual measurement values of the MEMS accelerometer; $g_{x}, g_{y}, g_{z}$ are the ideal measurement values of the MEMS accelerometer; $b_{x}, b_{y}, b_{z}$ are MEMS accelerometer bias; $a_{1}, a_{2}, a_{3}, a_{4}, a_{5}, a_{6}$ are installation error coefficients; $S_{X}, S_{y}, S_{z}$ are scale factors for the MEMS accelerometer; $n_{x}, n_{y}, n_{z}$ are random measurement noise. 


\section{The Six-position Static Calibration Method of MEMS Accelerometer}

For different error models,static calibration of the accelerometer uses different static position. In order to avoid the random measurement noise,each static position is sampled five times, interval $1 \mathrm{~s}$ and the measurement results are the average of five dates. Substituting the ideal measurement values into the equation (1), we have the following equation:

$\mathrm{X}$-axis ideal measurement values are

$$
\begin{array}{lll}
h_{x 1}=b_{x}-K_{12} & h_{x 2}=b_{x}-K_{13} & h_{x 3}=b_{x}+S_{x} \\
h_{x 4}=b_{x}+K_{12} & h_{x 5}=b_{x}-S_{x} & h_{x 6}=b_{x}+K_{13}
\end{array}
$$

Y-axis ideal measurement values are

$$
\begin{array}{lll}
h_{y 1}=b_{y}-S_{y} & h_{y 2}=b_{y}-K_{23} & h_{y 3}=b_{y}+K_{21} \\
h_{y 4}=b_{y}+S_{y} & h_{y 5}=b_{y}-K_{21} & h_{y 6}=b_{y}+K_{23}
\end{array}
$$

$\mathrm{Z}$-axis ideal measurement values are

$$
\begin{array}{ccc}
h_{z 1}=b_{z}-S_{y} & h_{z 2}=b_{z}-S_{z} & h_{z 3}=b_{z}+K_{31} \\
h_{z 4}=b_{z}+K_{32} & h_{z 5}=b_{z}-K_{31} & h_{z 6}=b_{z}+S_{z}
\end{array}
$$

According to equation (2), (3) (4), we have the following results:

$$
\begin{array}{llll}
4 b_{x}=h_{x 1}+h_{x 2}+h_{x 4}+h_{x 6} & 2 S_{x}=h_{x 3}-h_{x 5} & 2 K_{12}=h_{x 4}-h_{x 1} & 2 K_{13}=h_{x 6}-h_{x 2} \\
4 b_{y}=h_{y 2}+h_{y 3}+h_{y 5}+h_{y 6} & 2 S_{y}=h_{y 4}-h_{y 1} & 2 K_{21}=h_{y 3}-h_{y 5} & 2 K_{23}=h_{y 6}-h_{y 2} \\
4 b_{z}=h_{z 1}+h_{z 3}+h_{x 4}+h_{x 6} & 2 S_{z}=h_{z 6}-h_{z 2} & 2 K_{31}=h_{z 3}-h_{z 5} & 2 K_{32}=h_{z 4}-h_{z 1}
\end{array}
$$

Substitute the actual measurement values into the equation (5) and simplify the result, the coefficients of the error model are:

$$
\left[\begin{array}{l}
g_{x} \\
g_{y} \\
g_{z}
\end{array}\right]=\left[\begin{array}{ccc}
1.0000 & 0.0027 & -0.0058 \\
0.0052 & 1.0063 & 0.0153 \\
0.0013 & -0.0520 & 0.9942
\end{array}\right]\left[\begin{array}{c}
h_{x}+11.06 \\
h_{y}-0.20 \\
h_{z}-31.85
\end{array}\right]
$$

\section{Accelerometer static Calibration method based on the PSO algorithm}

The PSO algorithm is a stochastic, swarm intelligence based on global optimization algorithm, and has the advantages of fast convergence, easy to be implemented, a simple calculation model and insensitive to initial value. Analyzing a number of improved PSO algorithm, Daniel Bratton and James Kennedy proposed the standard PSO algorithm in $2007^{[5]}$.

When the accelerometer is in standstill, the accelerometer measurement is the acceleration of gravity. In this paper, $\left\|g_{\text {ref }}\right\|=9.8 \mathrm{~m} / \mathrm{s}^{2}$. The measurement value should be distributed in a sphere, centered at $(0,0,0)$, radius of $\left\|g_{\text {ref }}\right\|=9.8 \mathrm{~m} / \mathrm{s}^{2}$. Because of the measurement error,the actual measurement value is distributed in an ellipsoid. So the acceleration static calibration is the parameter estimation under the constraint of an ellipsoid. Therefore, the fitness function of the PSO algorithm is the mean square error between the reference valus $\left\|g_{\text {ref }}\right\|$ and the corresponding data vector $\left\|g_{n}\right\|$.

$$
O(p)=\frac{1}{N} \sum_{n=1}^{n=N}\left(\left\|g_{r e f}\right\|-\left\|g_{n}\right\|\right)^{2}, \quad\left[\begin{array}{l}
g_{n x} \\
g_{n y} \\
g_{n z}
\end{array}\right]=\left[\begin{array}{lll}
K_{11} & K_{12} & K_{13} \\
K_{21} & K_{22} & K_{23} \\
K_{31} & K_{32} & K_{33}
\end{array}\right]\left[\begin{array}{l}
h_{x}-b_{x} \\
h_{y}-b_{y} \\
h_{z}-b_{z}
\end{array}\right]
$$

Where $N$ is the sampling number, $g_{\text {ref }}$ is the reference acceleration of gravity, $\mathrm{h}$ is the actual measurement values, and $\mathrm{g}$ is the acceleration calibrated by static calibration method. From the above analysis, there are 12 parameters to be estimated. So the dimension of the particle is 12.According to the standard PSO algorithm, the error calibration model is as follows 


$$
\left[\begin{array}{l}
g_{x} \\
g_{y} \\
g_{z}
\end{array}\right]=\left[\begin{array}{ccc}
0.9871 & 0.0029 & -0.0144 \\
0.0751 & 0.9813 & 0.0548 \\
0.0243 & -0.0268 & 0.9803
\end{array}\right]\left[\begin{array}{c}
h_{x}+4.7816 \\
h_{y}-1.0140 \\
h_{z}-33.5546
\end{array}\right]
$$

\section{Analysis of Experimental Results}

Through the I2C bus, collect the original acceleration, and sample a total number of 1936 at $40 \mathrm{~Hz}$. According to the six-position and PSO static calibration method, calibrate the original acceleration respectively. Distribution of the acceleration's amplitude is shown in Figure 1.

From Figure 1, the amplitude of the acceleration is calibrated by the PSO static calibration method, distributed in $1 \mathrm{~g}$ nearby, and its calibration effect is better than of six position static calibration method. The standard deviation of the original acceleration's amplitude is $0.1098 \mathrm{~g}$. If calibrated by the PSO static calibration method, the standard deviation of the acceleration's amplitude is $0.036 \mathrm{~g}$. If calibrated by the six-position static calibration method, the standard deviation of the acceleration's amplitude is 0.0447g; and the standard deviation of the PSO static calibration method is $20 \%$ smaller than the standard deviation of the six-position static calibration method.
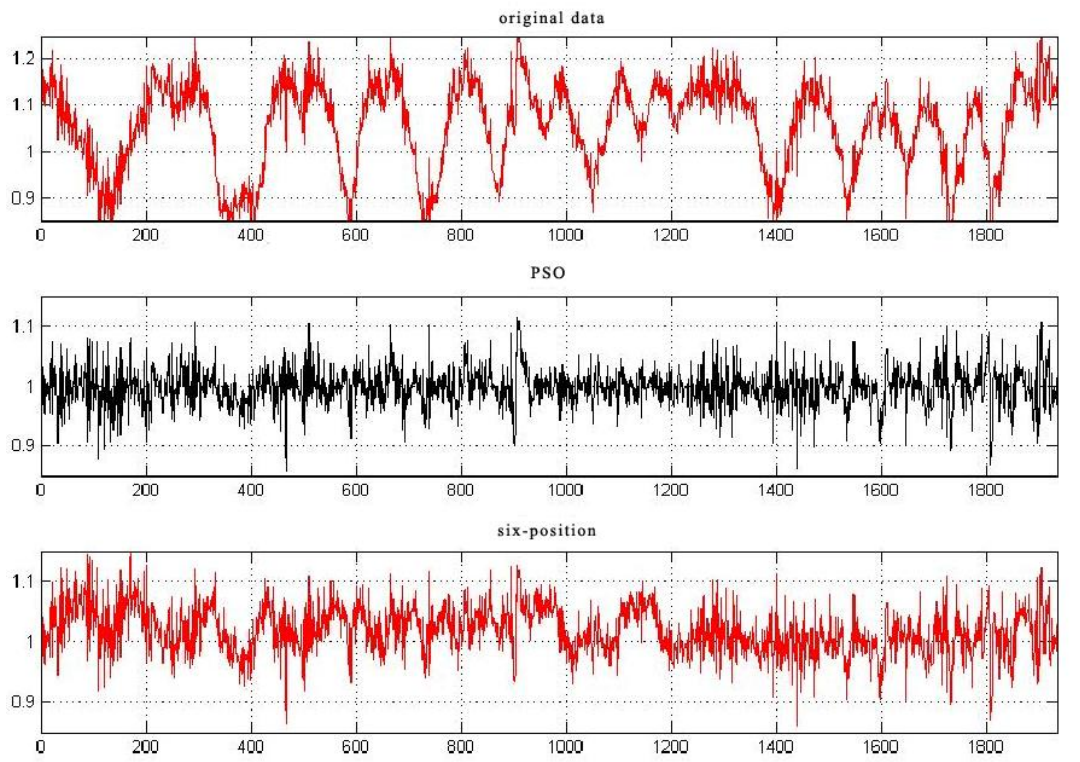

Fig.1 Amplitude distributing of the acceleration before-and-after calibration

According to the equation (6) and (8), the measurement values of X, Y, Z axes are calibrated, and the measurement standard deviation is shown in Table 1. It can be concluded that the measurement standard deviation of the PSO static calibration method has been significantly decreased, compared with the six-position static calibration method.

Table.1 Measurement standard deviation of all the axes

\begin{tabular}{|c|c|c|c|c|c|c|}
\hline \multirow{2}{*}{ axes pose } & \multicolumn{2}{|c|}{$\begin{array}{l}\theta=0 \\
\varphi=(-180 \sim 165)\end{array}$} & \multicolumn{2}{|c|}{$\begin{array}{l}\theta=(-75 \sim 75) \\
\varphi=0\end{array}$} & \multicolumn{2}{|c|}{$\begin{array}{l}\theta=(-75 \sim 75) \\
\varphi=-90\end{array}$} \\
\hline & PSO & six-position & PSO & six-position & PSO & six-position \\
\hline X & 10.23264 & 6.19166 & 3.471149859 & 4.836497 & 6.985309 & 5.276756 \\
\hline Y & 4.370645 & 11.08173 & 4.701516836 & 18.28473 & 5.843941 & 17.9379 \\
\hline$Z$ & 4.359236 & 7.240936 & 4.427102406 & 9.347633 & 9.086103 & 12.52955 \\
\hline
\end{tabular}

According to the above formula, the pitch angle is $\theta=a \tan (-X)$, the roll angle is $\varphi=a \tan (Y / Z)$. After calibrating the measurement values of $\mathrm{X}, \mathrm{Y}, \mathrm{Z}$ axes, calculate the accelerometer pitch and roll angle respectively. The angle error standard deviation is shown in Table 2. 
Table.2 Standard deviation of all the pose angles

\begin{tabular}{|c|c|c|c|c|c|c|}
\hline \multirow{2}{*}{ calibration } & \multicolumn{2}{c|}{$\begin{array}{c}\theta=0 \\
\varphi=(-180 \sim 165)\end{array}$} & \multicolumn{2}{c|}{$\begin{array}{c}\theta=(-75 \sim 75) \\
\varphi=0\end{array}$} & \multicolumn{2}{c|}{$\begin{array}{c}\theta=(-75 \sim 75) \\
\varphi=-90\end{array}$} \\
\cline { 2 - 7 } & $\theta$ & $\varphi$ & $\theta$ & $\varphi$ & $\theta$ & $\varphi$ \\
\hline $\begin{array}{c}\text { standard deviation } \\
\text { of PSO }\end{array}$ & 2.3026 & 1.2296 & 0.8431 & 1.8378 & 1.5014 & 2.3149 \\
\hline $\begin{array}{c}\text { standard deviation } \\
\text { of six-position }\end{array}$ & 1.4067 & 2.5368 & 0.9393 & 5.8723 & 2.6728 & 3.6797 \\
\hline
\end{tabular}

\section{Conclusions}

This paper proposes the Accelerometer static calibration method based on the PSO algorithm. The method is accurate, easy to operate. This paper compares the proposed method with the six-position static calibration method. The experimental results verify the validity of the method. Similar to other static calibration methods, the PSO static calibration method can not estimate the dynamic error of the accelerometer,so it is necessary to filter the acceleration measurement.

\section{References}

[1] Seong-hoon Peter Won, Farid Golnaraghi, A trail Accelerometer Calibration Method Using a Mathematical Model, IEEE TRANSACTUINS ON INSTRUMENTATION AND MEASUREMENT, VOL 59, NO.8,AUGUST 2010

[2] Vladimir Chapsky, Vladimir T. Portman, Ben-Zion Sandler,Single-mass 6-DOF isotropic accelerometer with segmented PSD sensors[J],Sensors and Actuators A: Physical, Volume 135, Issue 2, 15 April 2007, Pages 558-569

[3] Gustavo P. Ripper, Ronaldo S. Dias, Guilherme A. Garcia, Primary accelerometer calibration problems due to vibration exciters[J],Measurement, Volume 42, Issue 9, November 2009, Pages 1363-1369

[4] Akira Umeda, Mike Onoe, Kohji Sakata, Takehiro Fukushia, Kouichi Kanari, Hiroshi Iioka, Toshiyuki Kobayashi, Calibration of three-axis accelerometers using a three-dimensional vibration generator and three laser interferometers[J], Sensors and Actuators A: Physical, Volume 114, Issue 1, 20 August 2004, Pages 93-101

[5] Daniel Bratton, James Kennedy, Defining a Standard for Particle Swarm Optimization, Proceedings of the 2007 IEEE Swarm Intelligence Symposium (SIS 2007) 